\title{
Thrombolytic properties and side effects of brinase (fibrinolytic enzyme from Aspergillus oryzae) in the dog
}

\author{
WALTER H. E. ROSCHLAU \\ From the Department of Pharmacology, University of Toronto, Ontario, Canada
}

The thrombolytic properties of 'brinase' were investigated in the dog on standard 24-hour-old thrombi produced in the femoral artery and jugular vein by scarification of the intima (Roschlau, 1964). These thrombi measured about 1.5 to $2.5 \mathrm{~cm}$ adhering firmly to the intima and occluding the vessels as confirmed by zero flow and pressure downstream from the thrombus. They resembled in structure and composition those commonly associated with thromboembolic disease. Since spontaneous lysis did not occur within appropriate observation times, clot resolution after fibrinolytic treatment of the animals was ascribed to the direct effects of therapy.

\section{Systemic Administration}

Systemic intravenous infusions of 'brinase' were standardized to deliver $25000 \mathrm{C} 2$ units $(=2.5 \mathrm{mg})$ in $10 \mathrm{ml}$ of physiological saline per minute. Thrombolysis was assessed by direct observation and by the measurement of blood flow and pressure beyond the occlusion, followed after varying observation times by detailed necropsy studies and histopathological examinations.

The thrombolytic efficacy and safety of systemic brinase infusions were found to depend on the individual pretreatment inhibitor titre, which was termed 'protease resistance'. A quickly performed and simple inhibitor test was developed for bedside use. It employed a series of small test tubes containing a constant amount of thrombin and increasing amounts of brinase, with which $1 \mathrm{ml}$ aliquots of the animal's blood were allowed to react during a 10-minute incubation time. There was initial clotting, followed by lysis in those tubes whose concentration of brinase exceeded the inhibitor content of the blood. The end point was expressed as the number of units of brinase required to liquefy a $1 \mathrm{ml}$ blood clot within 10 minutes (reported as the 'test tube requirement' or TTR units). From the pretreatment TTR value a total systemic dose was calculated by a correlation table (Roschlau, 1964; Roschlau and Tosoni, 1965).
Since the mean protease resistance of a large dog population was 85 TTR units with a range from 50 to 120 TTR units, the inhibitor titre was always measured before treatment for the prediction of a systemic dose, and during an infusion for the monitoring of progressive inhibitor neutralization.

Occluding arterial and venous thrombi were rapidly lysed within a few hours following single infusions of doses of brinase that reduced protease resistance to 30 TTR units (Roschlau, 1964). Inhibitor recovery to pretreatment levels occurred within 24 hours following single brinase infusions. Sustained inhibitor reduction to 40 TTR units by repeated administrations of smaller enzyme doses over two to three days (Roschlau, 1965) resulted in somewhat slower but equally complete thrombolysis.

When examined at necropsy, formerly thrombosed vessel segments did not differ from non-thrombosed vessels, except for the lesions caused by scarification of the intima during thrombus production. All vessel segments examined for thrombus remnants which, when present, consisted of white platelet deposits without red clot. Long-term survival studies following brinase thrombolysis showed no rethrombosis in vessels bearing primary intima lesions, despite the intentional omission of anticoagulants during the posttreatment period.

Side effects were usually absent if protease resistance was prevented from falling below the $\mathbf{3 0}$ TTR units target level. However, intentional or accidental overdose with excessive reduction or depletion of inhibitors resulted in a number of untoward reactions that can be summarized as follows: (Roschlau and Tosoni, 1965; Roschlau, Freedman, and Miller, 1969; Freedman and Roschlau, 1970).

\section{Haematological}

A moderate transient elevation of whole blood clotting time was observed with all doses of brinase that exceeded the 30 TTR units threshold of protease resistance reduction. This was attributed to the 
action of fibrinogen degradation products. A reversible haemoconcentration was frequently noted, which seemed to be unrelated to the size of a given dose.

\section{Haemodynamic}

Slight hypotensive fluctuations occurred in the majority of cases, becoming progressively severe as brinase inhibitors were reduced. Intentional depletion of inhibitors was always accompanied by profound hypotension, which occasionally resulted in the death of the animal from acute cardiovascular collapse. The haemodynamic effects were attributed to the kininogenic properties of the proteolytic enzyme, leading to progressive activation of vasodepressor polypeptides.

\section{Systemic}

An elevation of liver enzymes (transaminases) was regularly associated with excessive brinase administration. This was considered to be an indication of free proteolysis following the depletion of brinase inhibitors. Animals showing high elevations of transaminase values during therapy usually displayed varying degrees of internal extravasation at necropsy.

The appearance of side effects was without exception related to the drug-induced depression of protective brinase inhibitors. In the dog, the critical level of protease resistance was found to be near 30 TTR units, at which level optimal thrombolytic activity was obtained without major side effects. We have therefore adopted the target of 30 TTR units as the non-toxic thrombolytic endpoint of inhibitor neutralization during systemic brinase therapy.

\section{Local Administration}

Local thrombolysis of artificial vascular occlusions in dogs was assessed by injecting brinase via a cannula directly to the thrombus head (Roschlau and Fisher, 1966). A solution containing $1000 \mathrm{C} 2$ units of brinase per $\mathrm{ml}$ of physiological saline was administered in $2 \mathrm{ml}$ aliquots in intervals of five to 10 minutes. Thrombolysis was assessed by saline filling of the vessel and transillumination. Following thrombolysis, the animals received systemic heparin in order to maintain an elevated clotting time for an additional 24 hours.

The first injection of 2000 units of brinase frequently caused additional clotting in the injected segment. Although subsequent doses of enzyme overbalanced this initial clot promotion, pretreatment with heparin (or the admixture of small amounts of heparin to the brinase solution) was desirable to obtain prompt lysis with minimal enzyme doses.

After pretreatment with heparin arterial and venous thrombi lysed within minutes of injection of brinase. Two doses of 2000 units usually canalized the thrombi, and two or three injections resulted in complete removal of thrombotic material. The duration of treatment rarely exceeded $\mathbf{3 0}$ minutes, the vessels remaining patent under moderate anticoagulation.

The instillation of small amounts of brinase into isolated vessel segments was entirely without systemic side effects. At necropsy 24 hours after brinase treatment, both arteries and veins were found to be free of thrombus, and the appearance of the vessels was essentially normal, excepting the scars at the primary thrombus site.

\section{Conclusions}

These experiments showed that systemic administration of brinase required the partial neutralization of circulating inhibitors, which exist in a variety of mammalian species including man. Inhibitor titres vary between species and between individuals. Prediction and titration of individual dosage, therefore, was recognized as a requisite for the attainment of optimal thrombolytic activity and the prevention of overdosage and side effects. Optimal inhibitor neutralization has been defined experimentally, and methods of dose prediction and monitoring of inhibitor levels have been developed.

The side effects that may occur from overtreatment with brinase appear all to be related to the progressive depletion of protective inhibitor substances. Provided that basic mechanisms of action of brinase are recognized and incorporated in the therapeutic approach, brinase may be used as a potent and safe systemic agent in clinical thrombolysis.

The doses required to effect thrombolysis with local administration were found to be substantially lower than systemic requirements. The effects on inhibitors were hardly perceptible, conferring to the local administration an unusually great margin of safety and obviating the laboratory control of systemic therapy. This has resulted in the adoption of brinase as the agent of choice for the treatment of clotting disorders during long-term haemodialysis therapy (Roschlau, 1968). 